\title{
Ruido ambiental en áreas verdes urbanas y periurbanas de una microcuenca en Heredia, Costa Rica
}

\author{
Danny Alfaro-Rojas $^{1}(\mathbb{D})$, lan Portuguez-Brenes ${ }^{1}(\mathbb{D})$, Héctor Perdomo- Velázquez ${ }^{(i D}$ \& Roberto Vargas - \\ Masis $^{3}$ (iD
}

1. Universidad Estatal a Distancia, Escuela de Ciencias Exactas y Naturales, Programa de Manejo de Recursos Naturales, Mercedes de Montes de Oca, San José, Costa Rica; danny.alfaro@uned.cr, arturitoportuguez@gmail.com

2. Universidad Nacional Autónoma de México, Centro de Estudios Mexicanos, San Pedro, San José, Costa Rica; hperdomo@unam.mx

3. Universidad Estatal a Distancia, Vicerrectoría de Investigación, Laboratorio de Investigación e Innovación Tecnológica, Sabanilla, San José, Costa Rica; rovargas@uned.ac.cr

Recibido 23-III-2020 C Corregido 31-VII-2020 • Aceptado 19-VIII-2020

DOI: $10.22458 /$ urj.v12i2.2846

\begin{abstract}
Environmental noise in urban and periurban green areas of a micro basin in Heredia, Costa Rica". Introduction: One of the manifestations of environmental impacts caused by urbanization is noise pollution, related to the presence of unwanted sound levels caused by human activities. These affects wildlife with discomfort, stress, and other harmful effects and might even include physical damage. Objective: To analyze the noise present in peri-urban and urban green areas adjacent to the micro basin of the Bermúdez River, in Heredia, Costa Rica. Methods: We installed Audiomoth automated recording equipment in six sampling sites along the micro basin. Three study sites were established in the peri-urban area and three in the urban area. Devices were programmed to record 1 minute every 10 minutes for 24 hours over 6 continuous days, twice per site. The Noise Index (SPL1) was used to evaluate the recorded sounds through sound pressure measurements. Results: The average noise levels in the urban area were significantly higher than in the peri-urban area $(\mathrm{V}=3383238, \mathrm{P}<0,001)$. We found significant differences in the six sampling sites $(F=3$ $773,65, P<0,001)$. Noise levels were different both day and night $(F=5578,21, P<0,001$ and $F=1472,09, P<0,001$, respectively). Conclusions: Higher noise levels occur in areas with a higher anthropic incidence, basically from urbanization, industry, construction, and transport, with a potential to affect the local wildlife.
\end{abstract}

Keywords: Environmental noise, ecoacoustics, urban ecology, micro-basin, audiomoth.
RESUMEN. Introducción: Una manifestación de los impactos ambientales provocados por la urbanización es la contaminación acústica, relacionada con la presencia de niveles sonoros no deseados provocados por actividades humanas. Estos afectan a la vida silvestre con incomodidad, estrés y otros efectos dañinos, e incluso pueden incluir daño físico. Objetivo: Analizar el ruido presente en áreas verdes periurbanas y urbanas adyacentes a la microcuenca del río Bermúdez, en Heredia, Costa Rica. Métodos: Instalamos equipos de grabación automatizados Audiomoth en seis sitios de muestreo a lo largo de la microcuenca. Se establecieron tres sitios de estudio en el área periurbana y tres en el área urbana. Los dispositivos se programaron para registrar 1 minuto cada 10 minutos durante 24 horas durante 6 días continuos, dos veces por sitio. El índice de ruido (SPL1) se utilizó para evaluar los sonidos grabados a través de medidas de presión sonora. Resultados: Los niveles de ruido promedio en el área urbana fueron significativamente más altos que en el área periurbana $(\mathrm{V}=3383238, \mathrm{P}<0,001)$. Encontramos diferencias significativas en los seis sitios de muestreo ( $F=3773,65$, $\mathrm{P}<0,001)$. Los niveles de ruido fueron diferentes tanto de día como de noche ( $F=5578,21, P<0,001$ y $F=1$ 472,09, $\mathrm{P}<0,001$, respectivamente). Conclusiones: Los niveles de ruido más altos se dan en áreas con mayor incidencia antrópica, básicamente de urbanización, industria, construcción y transporte, con potencial de afectar la vida silvestre local.

Palabras clave: Ruido ambiental, ecoacústica, ecología urbana, microcuenca, audiomoth. 
El desarrollo urbano en Costa Rica se concentra en mayor grado en la gran área metropolitana (GAM) (Ureña, Jiménez, Reynolds, \& Jones, 2005). Esta zona es el principal asentamiento urbano de Costa Rica, que integra las cuatro ciudades más grandes del país: San José, Alajuela, Cartago y Heredia, ubicadas en la Meseta Central. En Heredia, el aumento de la población ha generado un acelerado crecimiento del área urbana, lo que ha producido la fragmentación de áreas boscosas, la degradación de hábitats y la pérdida de diversidad biológica (Acosta, 2013).

Un área urbana representa un paisaje propio de los núcleos urbanos que se caracteriza por su alta densidad de población, extensión e infraestructura, pero principalmente las funcionalidades económicas y de empleo para los diferentes sectores productivos (Salinas \& Pérez, 2011). La zona periurbana se puede considerar como una zona de transición entre lo natural y lo "artificial", un área de contacto entre ecosistemas y como un mosaico de relictos naturales que coexisten con agroecosistemas y aglomeraciones urbanas que proporcionan variados servicios ecosistémicos (PRUGAM, 2015).

El sonido es una parte integral de los ecosistemas, ya que evidencia la complejidad en la comunicación de algunos seres vivos como información indispensable para sobrevivir y reproducirse (Sueur, Farina, Gasc, Pieretti, \& Pavoine, 2014). En contraste, el ruido se relaciona con la presencia en el ambiente de niveles sonoros no deseados y molestos y es característico de ambientes urbanos. Los estudios sobre ruido ambiental se basan en el estudio o la comprensión de cómo el sonido desde sus diversas fuentes (geofísica, biológica y antropogénicas), afecta las dinámicas del paisaje a través del tiempo y el espacio (Pijanowski, Farina, Gage, Dumyahn, \& Krause, 2011).

Las geofonías se definen como los sonidos provenientes de un ambiente geofísico, entre estos están los originados por la lluvia, el viento, el correr del agua en un río, un trueno. Las biofonías son los sonidos emitidos por organismos en un espacio y tiempo específico, como un ave, anfibio, insecto, mamífero, etc. Las antropofonías son los sonidos producidos por objetos creados por el ser humano, por ejemplo, un automóvil, un avión, un equipo de sonido, una campana, etc., y son característicos de ambientes urbanos. (Pijanowski et al., 2011)

En ambientes naturales la comunicación acústica es vital y algunos animales utilizan vocalizaciones para el cortejo y apareamiento, defender un territorio o advertir la presencia de un depredador. El ruido puede traer consecuencias en la comunicación de la vida silvestre, ya que las perturbaciones acústicas provenientes de antropofonías, enmascaran las señales acústicas producidas por el emisor haciendo que el receptor no reciba el mensaje o lo discrimine (Nemeth \& Brumm, 2010).

El ruido es considerado un peligro para la salud humana y sus efectos son catalogados como un problema sanitario cada vez más agravante. Puede causar desde insomnio, ansiedad, depresión, estrés, entre otros efectos psicológicos, hasta la pérdida parcial o total de la audición. Un ambiente que sobrepase los 55 decibelios ( $\mathrm{dB}$ ) es considerado un ambiente ruidoso, de 75 a $100 \mathrm{~dB}$ es considerado un ambiente con ruido fuerte y superior a los $100 \mathrm{~dB}$ es considerado un ambiente con ruido intolerable (Amable et al., 2017).

Recientemente, se han desarrollado índices acústicos que mediante cálculos estadísticos permiten analizar la estructura y distribución de la energía acústica biológica y antrópica en las grabaciones (Towsey, Wimmer, Williamson, \& Roe, 2014). Sin embargo, los algoritmos existentes sobreestiman o subestiman las medidas de actividad biótica en presencia del ruido urbano, por lo que se han desarrollado otras métricas alternativas (Ramírez-Alán, 2019; Fairbrass, Rennett, Williams, Titheridge, \& Jones, 2017; Fuller, Axel, Tucker, \& Gage, 2015; Gasc, Pavoine, Lellouch, Grandcolas, \& Sueur, 2015; Sueur et al., 2014; Towsey et al., 2014). Los índices acústicos evalúan y caracterizan el paisaje acústico, y permiten interpretar la salud y los procesos ecológicos de los ecosistemas (Pieretti et al, 2015). 
Los patrones naturales del paisaje sonoro se ven reflejados en los patrones acústicos espaciales y temporales, y varían con ciertos gradientes ecológicos y de perturbación humana, entre ellos el uso de suelo y la estructura del hábitat (Pijanowski et al., 2011). Los estudios de ruido reflejan que los niveles de contaminación acústica aumentan en paisajes dominados por la actividad humana, es decir, en hábitats naturales más complejos y con menor perturbación antropogénica los niveles de ruido serán menores y con un incremento en los sonidos biológicos y geofísicos (Pijanowski et al., 2011).

El presente estudio tiene el objetivo de analizar y comparar los niveles de ruido en los sitios de estudio seleccionados, los cuales son áreas verdes urbanas y periurbanas asociadas a la microcuenca del río Bermúdez en Heredia, Costa Rica, con el fin de interpretar el comportamiento del ruido en el transcurso del día, la noche y sobre las veinticuatro horas; generando una línea base de investigación sobre el estudio de ruido, así como el uso de tecnologías emergentes para el estudio áreas urbanas.

\section{MATERIALES Y MÉTODOS}

Área de estudio: La microcuenca del río Bermúdez (Figura 1) se encuentra dentro de la provincia de Heredia en la GAM y; posee una extensión de $74,11 \mathrm{Km}^{2}$, Inicia con una elevación máxima de 1900 m.s.n.m. en el cantón de San Rafael y recorre los cantones de San Pablo, San Isidro, Santo Domingo, Barva, Heredia, Flores, Belén, en donde se une a la Cuenca del río Virilla en la provincia de Alajuela con una elevación de 800m.s.n.m. (Hernando \& Orozco, 2015).

Se determinaron sitios de muestreo en áreas verdes periurbanas y urbanas en las cercanías de la microcuenca del río Bermúdez, por su fácil accesibilidad, seguridad, y de acuerdo con diferentes características a nivel de cobertura vegetal, uso de suelo. Se eligieron tres áreas verdes periurbanas (Laguna San Rafael, Monte de la Cruz, Castillo Country Club) y tres áreas verdes urbanas (Parque del Norte, Polideportivo Santo Domingo, Cariari Country Club), que son utilizadas como áreas deportivas, recreativas, turísticas, vecinales.

Recolección de datos: Para las grabaciones se utilizaron dispositivos de grabación automática Audiomoth (Hill et al., 2017), que permiten hacer grabaciones con un amplio rango de frecuencias y ganancia. Se colocó un dispositivo en cada área de estudio, en un árbol, a una altura de $2 \mathrm{~m}$ desde el nivel del suelo. Los dispositivos se protegieron con una bolsa y un estuche plástico. Las grabaciones se realizaron en formato digital ".wav", a una frecuencia de muestreo de $48 \mathrm{kHz}$, 16 bits de resolución y ganancia media $(30,6 \mathrm{~dB})$ (Deichmann, Hernández-Serna, Delgado, CamposCerqueira, \& Mitchell, 2017), para captar el mayor rango posible de sonidos en el ambiente (Farina et al., 2014).

Las grabaciones se realizaron en cada sitio durante una semana de manera continua mediante 2 muestreos en cada sitio entre los meses de febrero y julio de 2019. Las grabadoras fueron configuradas para grabar 1 minuto en intervalos de 10 minutos durante las 24 horas del día (Deichmann et al., 2017). Se realizaron comparaciones por sitio de muestreo, por zona (urbana y periurbana) y por periodo temporal (día y noche). En los seis sitios de muestreo se generaron un total 14614 grabaciones de 1 minuto.

Análisis de datos: Para estandarizar el número total de grabaciones analizadas para cada sitio, se utilizaron 1822 para cada sitio, de acuerdo con la fecha de muestreo y seleccionando aquellas en el mayor rango temporal posible, obteniendo un total de 10932 grabaciones.

Los audios fueron procesados usando el paquete Sinax, con la función spl1() para obtener

el índice acústico de ruido. Esta función evalúa el nivel promedio de presión sonora (SPL) en las 
grabaciones de un minuto (Ramírez-Alán, 2019), el ruido se calcula en un intervalo temporal preciso t1- t2.

$$
L(E, T)=10 \log 10\left(\int_{t 1}^{t 2} \frac{p^{2}(t)}{p 0^{2}}\right)=L e q+10 \log 10^{\frac{L e q, 1}{10}}
$$

El nivel de presión de sonido $(S P L)$ se utiliza para evaluar de manera indirecta y de forma relativa el "ruido" ambiental y la evaluación urbana de la calidad del entorno sonoro (Ramírez-Alán, 2019; Farina, 2013). Se utilizó el lenguaje de programación R versión 3.6.1 (R Core Team, 2019) para este proceso.

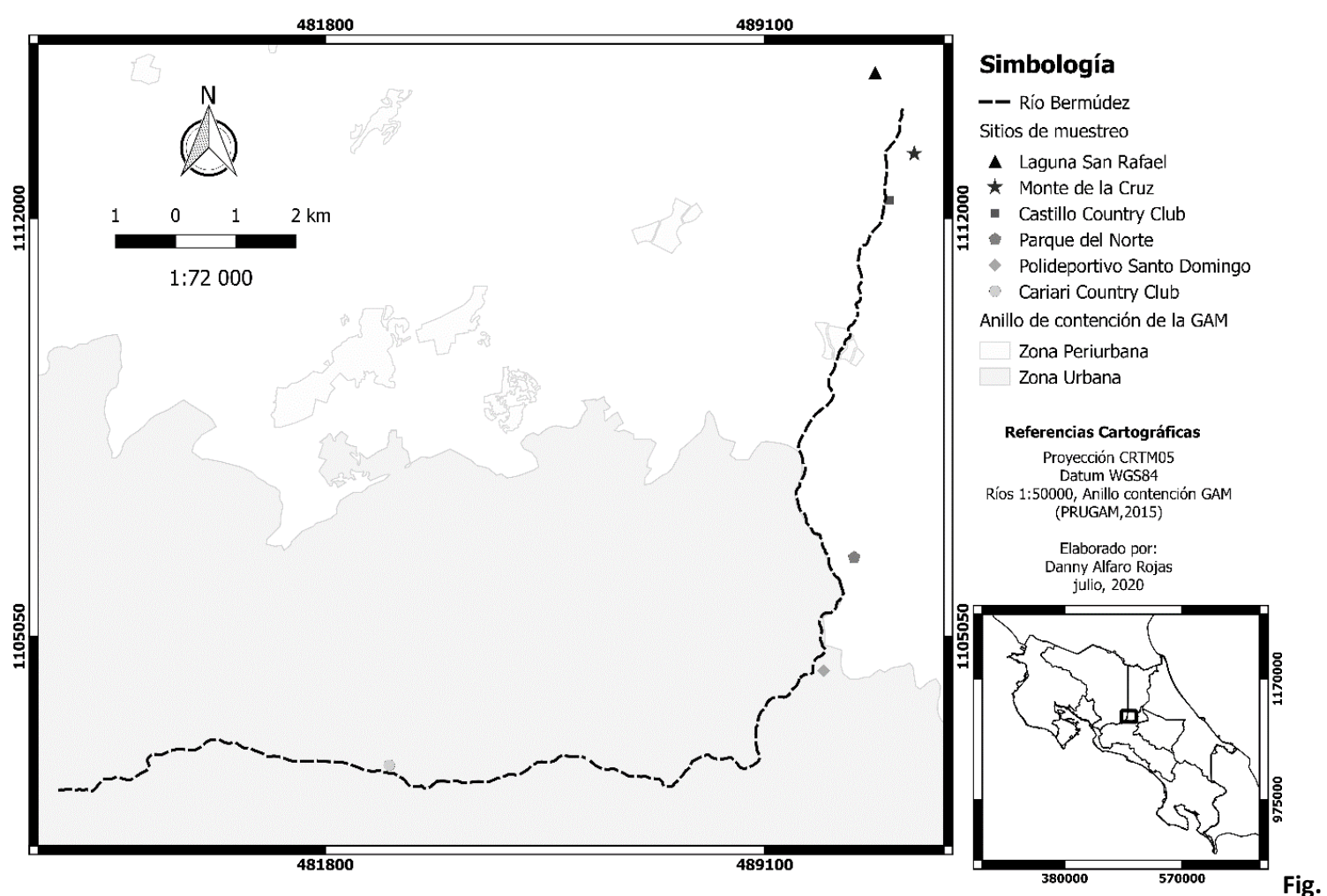

1. Mapa de ubicación de los 6 sitios de muestreo en la microcuenca del río Bermúdez - Heredia, 3 en la zona periurbana (Laguna San Rafael, Monte de la Cruz y Castillo Country Club) y 3 en la zona urbana (Parque del Norte, Polideportivo Santo Domingo y Cariari Country Club).

La herramienta sp/1, se encuentra en una versión de prueba por lo que fue necesario realizar un ajuste a los valores obtenidos. Para dicho ajuste se realizaron grabaciones en un ambiente controlado con un Audiomoth configurado en el mismo formato descrito anteriormente. Simultáneamente se recolectaron tres valores de ruido utilizando un sonómetro Extech 407730, configurado para captar el ruido $\mathrm{dB}(\mathrm{A})$, ambos equipos se colocaron a la misma altura y distancia de la fuente del sonido. Para estos valores se calculó el promedio para dividirlo entre el valor de ruido promedio exportado por el paquete Sinax en su herramienta spl1 y establecer el valor de ajuste (VA) en 1.4, considerando pruebas realizadas en campo. La función utilizada para dicho ajuste es la siguiente:

$$
V A=\frac{a_{1}+a_{2}+a_{3} \ldots a n}{n}
$$


Donde:

$$
a 1=\frac{(s p l 1)_{1}}{(v p 1)_{1}}
$$

Siendo $v p 1$ un valor correspondiente de $72,8 \mathrm{~dB}$ interpretado como el valor promedio de ruido de las pruebas realizadas con el sonómetro Extech 407730.

La media del índice de ruido de cada audio en las dos zonas de estudio periurbano y urbano fue evaluada mediante una prueba de hipótesis utilizando la prueba de Wilcoxon. Los seis sitios de estudio se evaluaron mediante una prueba robusta, en la cual se utilizó una media truncada y el ajuste de Welch (Mair \& Wilcox, 2020; Ramalle-Gómara \& Andrés de Llano, 2003), así como una comparación posteriori Lincon (Mair \& Wilcox, 2020).

Los sitios se evaluaron por separado en horas de día (6:00 horas a 18:00 horas) y horas de noche (18:00 horas a 6:00 horas.) Cada grupo se evaluó mediante una prueba robusta mediante una media truncada y el ajuste de Welch (Mair \& Wilcox, 2020; Ramalle-Gómara \& Andrés de Llano, 2003), y una comparación posteriori Lincoln (Mair \& Wilcox, 2020) la cual utiliza el ajuste de Welch (Welch, 1951). Por último, se realizó una comparación visual del comportamiento del índice de ruido respecto al tiempo en cada sitio de estudio. Para ello se calculó un promedio del índice de ruido de los datos que se encontraban dentro de cada hora. Todos los análisis estadísticos se realizaron con el lenguaje de programación R versión 4.0.2 (R CoreTeam, 2020) con un nivel de significancia de $\alpha=0,05$ y sin diferenciar las semanas de muestreo.

\section{RESULTADOS}

El índice de ruido fue significativamente más alto en la zona urbana que en la zona periurbana ( $\mathrm{V}=3383238, \mathrm{gl}=5465, \mathrm{P}<0,001$ ) con valores promedio de $68 \mathrm{~dB}$ en la zona urbana y $64 \mathrm{~dB}$ en la zona periurbana. El valor más alto de ruido de la zona urbana sobrepasa los $85 \mathrm{~dB}$ mientras el valor más alto de la zona periurbana alcanza $84 \mathrm{~dB}$, siendo este un valor atípico. Los valores mínimos son de $57 \mathrm{~dB}$ en promedio en ambas zonas, a pesar de ello, tanto la mediana como la media de la zona Urbana se encuentran muy por encima en comparación con la zona Periurbana (Fig. 2), demostrando que la zona de mayor incidencia antrópica posee niveles de ruido más altos.

En la figura tres (Fig. 3) se muestra el índice de ruido promedio de los seis sitios de muestreo, indica que el punto con mayor ruido es el polideportivo de Santo Domingo, ubicado en la zona urbana, con una media de 73dB. Los sitios de muestreo más alejado de la zona urbana, la laguna de San Rafael y el Monte de la Cruz poseen la menor cantidad de ruido promedio con $62 \mathrm{~dB}$ y $64 \mathrm{~dB}$ respectivamente, los tres sitios restantes tienen una composición muy similar. La prueba estadística robusta demostró diferencias significativas entre los sitios de estudio ( $F=3773,65, \mathrm{gl}=2$ 835,39, $\mathrm{P}$ $<0,001)$ y la prueba de comparación posteriori Lincon, demostró que había diferencias significativas entre todos los sitios (Lincon, $\mathrm{P}<0,001$ ). 


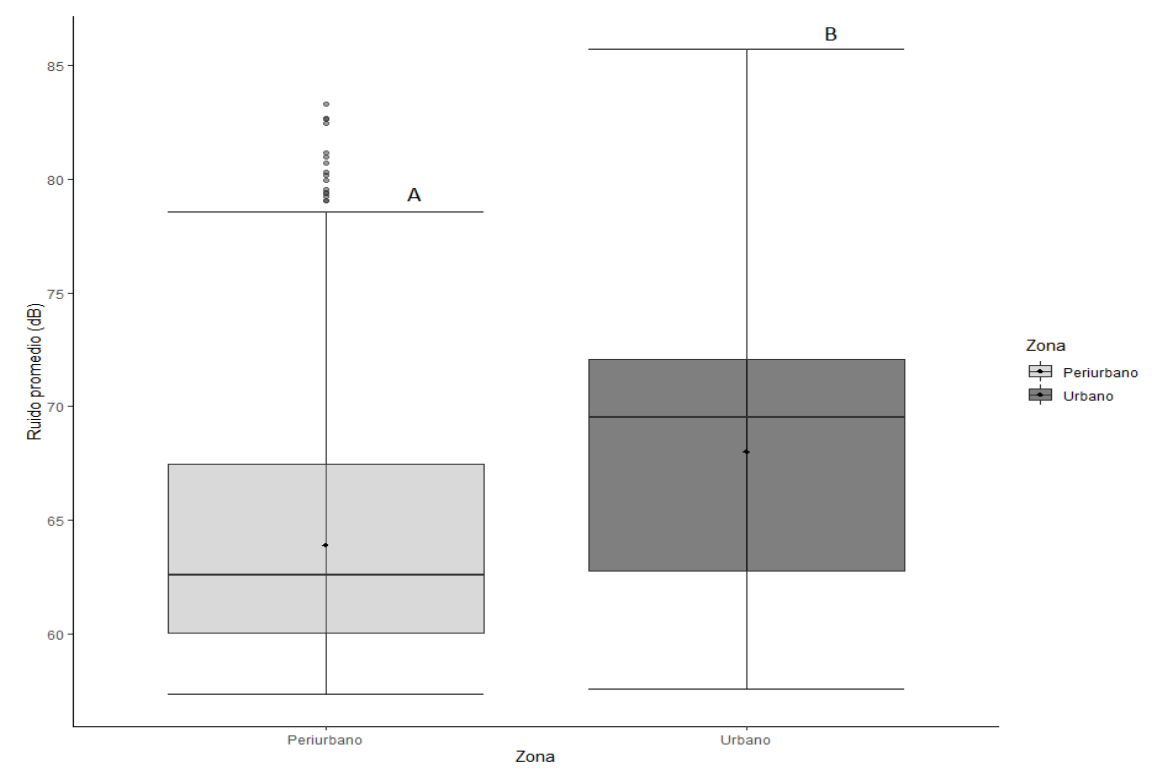

Fig. 2. Índice de ruido promedio de las dos zonas de muestreo. La línea horizontal, el punto dentro de la caja y los puntos por encima de esta, señalan la media, la mediana y los datos atípicos de cada conjunto de datos respectivamente. Letras iguales indican que no hay diferencias estadísticamente significativas entre los sitios de estudio $(P>0,05)$.

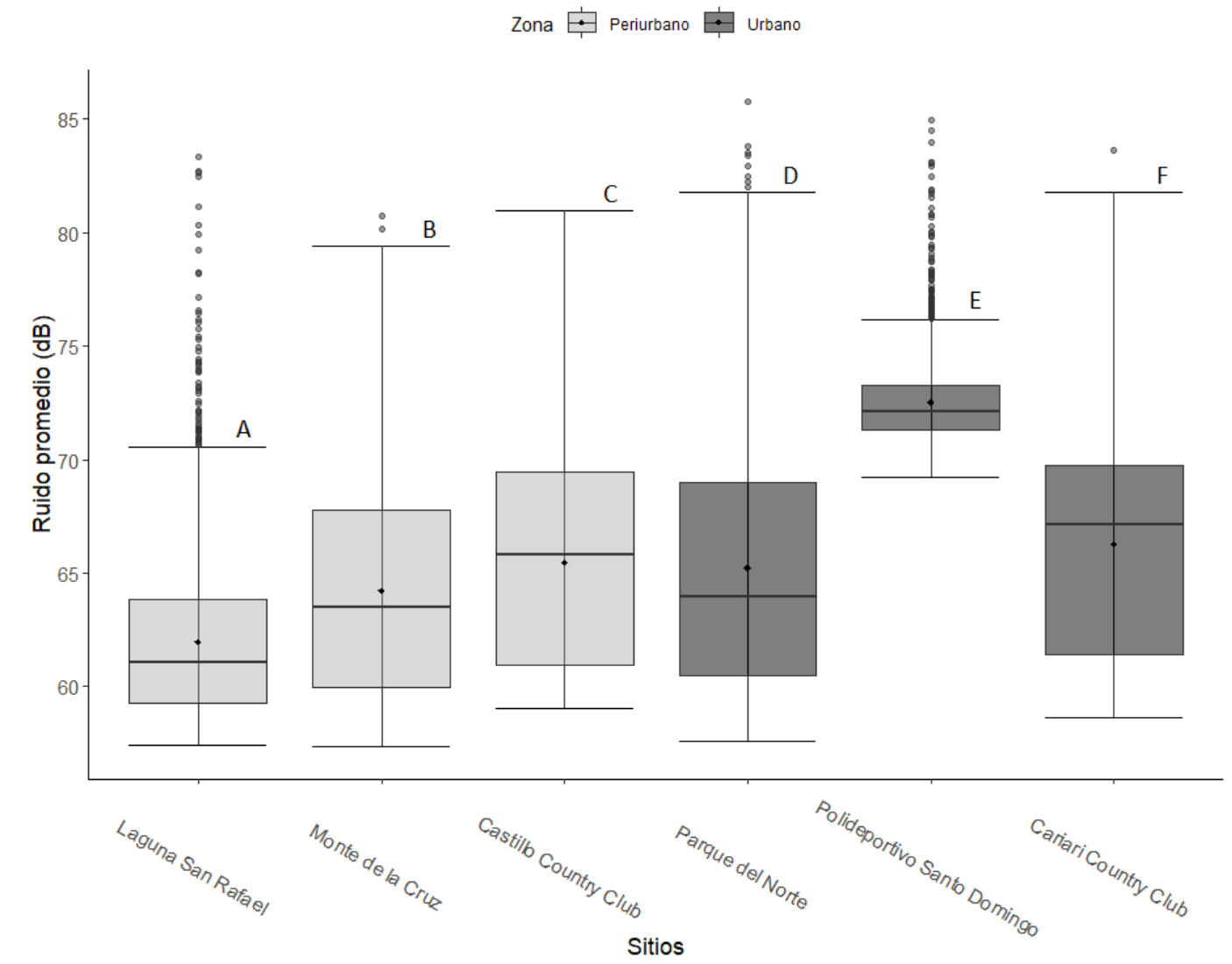

Fig. 3. Índice de ruido promedio de los seis sitios de estudio. La línea horizontal, el punto dentro de la caja y los puntos por encima de esta, señalan la media, la mediana y los datos atípicos de cada conjunto de datos respectivamente. Letras iguales indican que no hay diferencias estadísticamente significativas entre los sitios de estudio $(P>0,05)$. 
Al evaluar el índice de ruido promedio entre los sitios diferenciado en horas día y horas noches, se obtuvieron diferencias significativas en ambos casos: $F=5$ 578,21, gl=1 517,78, $P<0,001$ para el día y $\mathrm{F}=1472,094, \mathrm{gl}=1364,08, \mathrm{P}<0,001$ para la noche. La prueba de comparación posteriori Lincon indica que durante el día los sitios más parecidos son el Castillo Country Club y el Parque del Norte, a pesar de esto, existen diferencias significativas entre estos sitios al igual que entre todos los sitios restantes (Lincoln, $\mathrm{P}<0,01$ ). La misma prueba indica que durante la noche no existen diferencias significativas entre Cariari Country Club y Castillo Country Club $(P>0,05)$; sin embargo, en todas las comparaciones restantes entre sitios si existen diferencias significativas (Lincoln, $P<0,001)$.

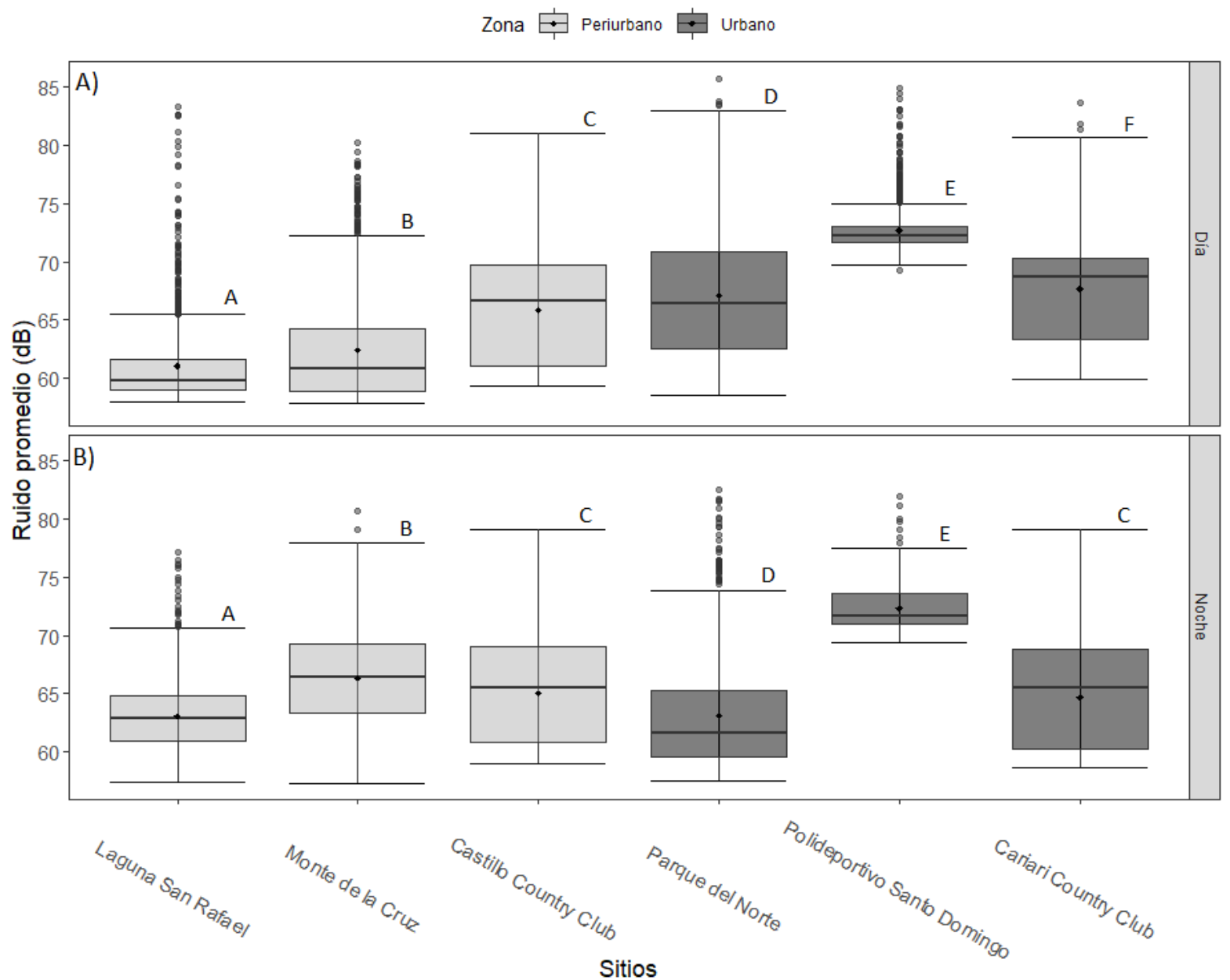

Fig. 4. A) Índice de ruido promedio de los seis sitios de estudio, dividido en horas día (06:00 horas a las 18:00 horas), B) Índice de ruido promedio de los seis sitios de estudio horas de noche (18:00 horas a las 06:00 horas). La línea horizontal, el punto dentro de la caja y los puntos por encima de esta, señalan la media, la mediana y los datos atípicos de cada conjunto de datos respectivamente. Letras iguales indican que no hay diferencias estadísticamente significativas entre los sitios de estudio $(P>0,05)$.

Con respecto al comportamiento del índice de ruido promedio durante las 24 horas del día se destaca el sitio menos cambiante y que presentó el índice de ruido promedio más alto con 74 $(\mathrm{dB})$ a las cuatro horas fue el Polideportivo de Santo Domingo (Fig. 5). Tanto la laguna de San Rafael como el Monte de la Cruz presentaron los valores más bajos en mayor cantidad de horas respecto a los otros sitios, siendo a las cuatro horas en la laguna de San Rafael con 59,50dB el valor más bajo de los seis sitios. 
El Monte de la Cruz a pesar de sus bajos valores a partir de las 11 horas, comienza a aumentar hasta llegar a los $69 \mathrm{~dB}$ siendo este el segundo valor más alto, excluyendo los valores del Polideportivo de Santo Domingo. Con base a esta misma exclusión, el valor más alto lo presenta el Parque del norte a las 10 horas con $70 \mathrm{~dB}$; el mismo sitio presenta una curva notoria a partir de las tres horas con $69 \mathrm{~dB}$ punto, donde hay un aumento constante entre horas hasta llegar a las 10 horas (punto más alto) y a partir de ese punto, se observa una reducción constante hasta llegar a las 17 horas. Tanto el Cariari Country Club como el Castillo Country Club no presentan fuertes fluctuaciones en comparación con otros sitios; sin embargo, se observan algunos cambios de hora a hora a partir de las nueve horas hasta las 19 horas.

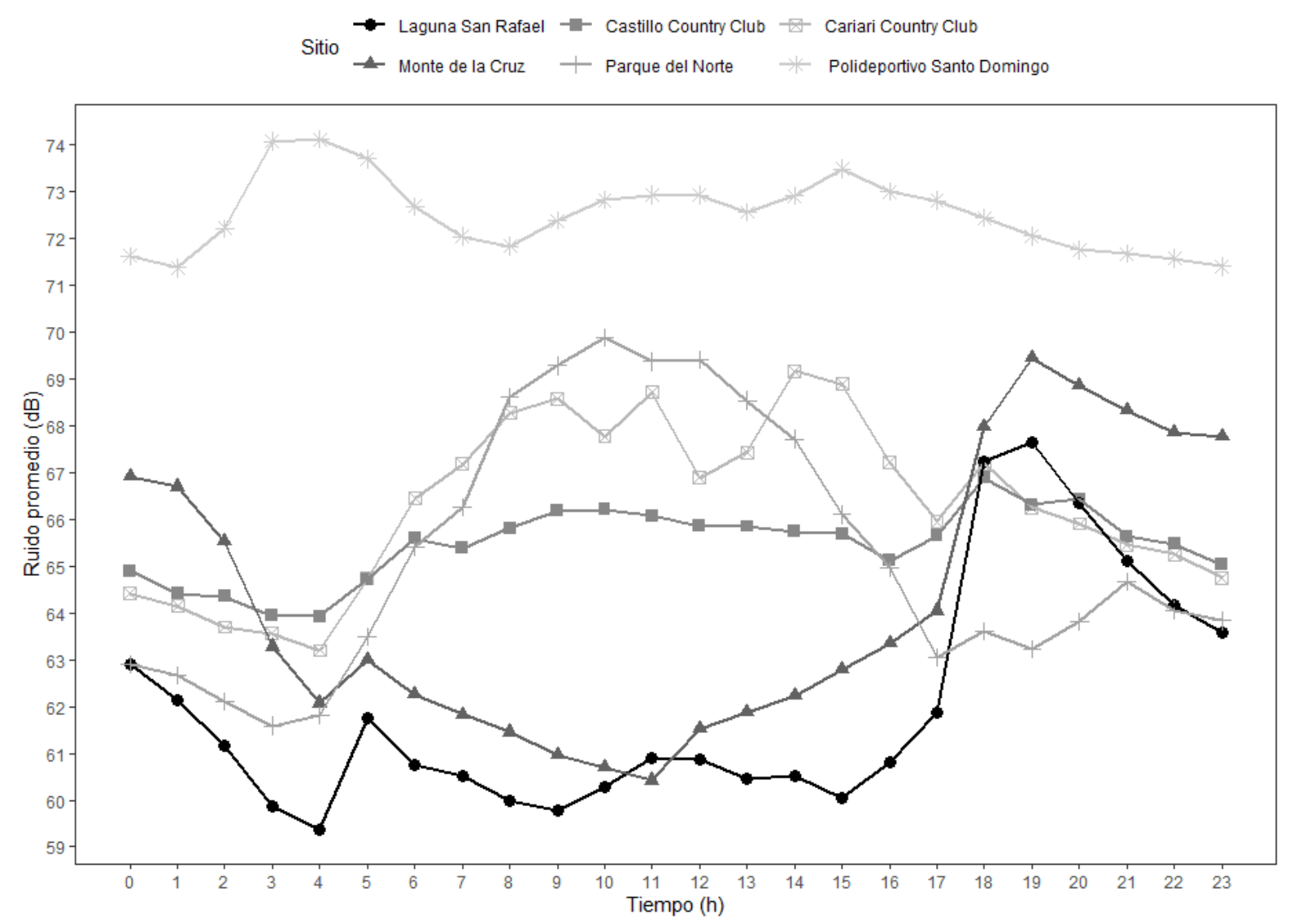

Fig. 5. La media del índice de ruido promedio por hora en cada sitio de estudio. Redondeo con dos decimales.

\section{DISCUSIÓN}

Factores como la urbanización, la industria, las construcciones, el transporte, entre otros, generan una alteración de los sonidos naturales. Estas actividades antrópicas son las causantes de altos valores de ruido generados en las zonas urbanizadas, siendo una de las principales fuentes de contaminación auditiva (Cohen \& Castillo, 2017). Los resultados obtenidos en el presente estudio indican que los sitios de la zona urbana tienen los niveles más altos de ruido.

El valor promedio del índice de ruido en la zona urbana osciló entre los 68 a 69dB, por lo tanto, es considerado como un ambiente ruidoso (Ministerio de Salud, 2000). Sin embargo, los valores más altos (mayores a $85 \mathrm{~dB}$ ) alcanzados en las grabaciones de esta zona ya son considerado como un ruido fuerte, pero sin alcanzar valores de ruido intolerable que se dan a partir de los $100 \mathrm{~dB}$ 
(Amable et al., 2017). Por ello, consideramos que en la zona urbana estudiada se mantiene en el rango de ruido tolerable, [A1] con importantes picos de ruidos fuertes.

Las diferencias significativas que se presentan entre todos los seis sitios de estudio se pueden deber a que los valores de ruido en áreas urbanas y periurbanas presentan una variabilidad espacio-temporal que se relacionan con las características del paisaje y con las actividades que el ser humano desarrolla (Liu, Kang, Luo, Behm, \& Coppack, 2013). Los ambientes urbanos con mayor densidad poblacional y mayor tráfico vehicular, presentan niveles sonoros más altos (Jeon \& Hong, 2015); por su parte, Pijanowski et al. (2011) mencionan que a medida que disminuye la actividad humana, disminuyen las antropofonías, áreas con variabilidad en el uso de suelo (agropecuario, bosque, residencial) como la zonas periurbanas, presentan mayor variabilidad acústica y menores niveles de presión sonora, concordando con los resultados obtenidos.

Por su parte, para el índice de ruido en el día y en la noche observamos que mantiene un patrón similar y que todos los sitios, tanto de día como de noche, presentan diferencias significativas, a excepción de Cariari Country Club y Castillo Country Club en horas de la noche. En una investigación similar utilizando índices acústicos, Do Nascimento, Campos-Cerqueira y Beard (2020) observaron que sus áreas de estudio mostraron valores acústicos diferentes tanto de día como de noche, y mencionan la capacidad de los índices acústicos para determinar la características y patrones acústicos de cada hábitat. Otros autores mencionan que las diferencias entre el día y la noche varían entre región, hábitat y componentes del paisaje sonoro (Bradfer-Lawrence et al., 2019; Fuller et al., 2015; Pieretti et al., 2015).

Con respecto a la gráfica de las 24 horas del día, observamos que los sitios periurbanos más alejados de la matriz urbana, de las 5 horas y hasta las 17 horas presentan un comportamiento similar que oscila en un nivel de ruido de los 59 a los $63 \mathrm{~dB}$ y a partir de las 18 horas tienen un incremento en los niveles de ruido. En los sitios más cercanos a la matriz urbana se observa lo opuesto, niveles de ruido alto de las 5 horas a las 17 horas que oscilan entre los 65 a los $70 \mathrm{~dB}$ con un descenso de las 18 horas a las 4 horas. En las zonas urbanas las actividades comerciales y el incremento de la cantidad de vehículos son factores que influyen en las altas tasas de ruido, y se considera que en estos sitios un aumento en la cobertura de vegetación puede beneficiar la reducción de ruido en estas áreas ya que sirve como un escudo protector (Pandya, 2001).

La estructura del sonido, las características y el comportamiento se relacionan con la estructura y composición del paisaje (Pijanowski et al., 2011). Joo, Gage y Kasten (2011) indican que los sonidos antropogénicos aumentan conforme se pasa del ambiente rural al ambiente urbano, y mencionan que el aumento de los niveles de ruido ambiental se relaciona con la proximidad al centro urbano. La zona periurbana conserva áreas naturales en las que habitan diversas especies, en estas zonas los sonidos nocturnos en su mayoría provienen de biofonías (insectos, anuros) (Farina, 2013), por lo que el incremento de ruido en los sitios más alejados de la matriz urbana a partir de las 18 horas se relaciona a esta situación.

Matsinos et al. (2008) mencionan que en áreas con actividades humanas hubo mayor incidencia de antropofonías de las seis horas a las 18 horas y en áreas donde converge lo natural con la actividad humana la incidencia de los sonidos proviene de biofonías, geofonías y en menor caso de antropofonías, concordando con nuestro estudio, donde en la misma franja horaria los sitios más cercanos a la matriz urbana presentan mayor ruido y los más alejados menor ocurrencia de ruido.

Los niveles de ruido en los sitios y zonas evaluadas superan los $55 \mathrm{~dB}$, siendo áreas con un ambiente ruidoso (Amable et al., 2017), es conocido que el ruido tiene efectos en la salud humana. Oro (2017) evidencia los problemas en la salud que el ser humano puede presentar, algunos de estos son: jaquecas, problemas cardiovasculares, digestivos, neurológicos, alteraciones del sueño, daños auditivos; por tanto, afectan a nivel auditivo, fisiológico y psicológico. 
La vida silvestre también es afectada por los altos niveles de ruido lo cual se evidencia en el estudio de Shannon et al. (2016) en el que recopilaron información de investigaciones entre 1990 al 2013, y concluyeron que el ruido es una amenaza para las especies y los ecosistemas a niveles de ruido de aproximadamente $40 \mathrm{~dB}$; estos estudios muestran que la vida silvestre altera su comportamiento vocal en presencia de hábitats ruidosos, cambia su comportamiento de vigilancia y forrajeo, y de manera general afecta la aptitud individual y la estructura de las poblaciones y comunidades.

Las áreas verdes urbanas y periurbanas proporcionan gran cantidad de servicios ecosistémicos para el bienestar humano tanto en la salud mental y física de los ciudadanos y pueden reducir el impacto de los niveles de ruido y ser sitios con las condiciones ambientales para el desarrollo de la vida silvestre y para mejorar la calidad de vida del ser humano (Sorensen, Barzetti, Keipi, \& Williams, 1998), una adecuada gestión y planificación urbana, y un manejo integral de las áreas verdes pueden mejorar la calidad ambiental de los sistemas urbanos donde vivimos.

En las ciudades convergen sonidos producidos por diferentes organismos vivos, fenómenos geofísicos y; ruidos antrópicos (Bustos, 2013). En los ambientes urbanos el ruido presenta una oportunidad para realizar estudios ecológicos, estudiar sus propiedades, funciones y la evolución del ambiente; y el efecto sobre los organismos que se encuentran bajo las presiones de esta variable sonora (Pavan, Scaravelli, Favaretto, \& Macchio, 2015).

Los estudios acústicos tienen un gran potencial para medir las condiciones ecológicas de los ambientes urbanos (Fairbrass et al., 2017; Joo et al., 2011), los estudios espacio-temporales en ecosistemas urbanos deben ser ampliados y dirigidos a evidenciar la necesidad de una mejor planificación urbana que tome en cuenta la naturaleza y la salud humana, así, los estudios acústicos serán una herramienta para modelar las ciudades del futuro.

\section{AGRADECIMIENTOS}

Al Laboratorio de Investigación e Innovación Tecnológica (LIIT) de la Universidad Estatal a Distancia (UNED) por brindarnos las herramientas necesarias para realizar la investigación. A las municipalidades de San Rafael de Heredia y de San Pablo de Heredia, al Comité de Deportes de Santo Domingo de Heredia, al Castillo Country Club y al Cariari Country Club que nos permitieron acceder a las áreas utilizadas como sitios de estudio.

\section{ÉTICA, CONFLICTO DE INTERESES Y DECLARACIÓN DE FINANCIAMIENTO}

Los autores declaran haber cumplido con todos los requisitos éticos y legales pertinentes, tanto durante el estudio como en el manuscrito; que no hay conflictos de interés de ningún tipo, y que todas las fuentes financieras se detallan plena y claramente en la sección de agradecimientos. Asimismo, están de acuerdo con la versión editada final del documento. El respectivo documento legal firmado se encuentra en los archivos de la revista.

La declaración de la contribución de cada autor al manuscrito es la siguiente: D.A.R.: investigador, análisis de datos y redactor del estudio, redacción del artículo. I.P.B.: análisis de datos y redacción del artículo. H.P.B: director del estudio, redacción del artículo. R.V.M.: director del estudio, redacción del artículo. 


\section{REFERENCIAS}

Acosta, V. (2013). Pérdida de hábitats y biodiversidad desvanecida en la ciudad de Heredia (Costa Rica). Ambientico, 41(3), 64-74

Amable, I., Méndez, J., Delgado, L., Acebo, F., de Armas, J., \& Rivero, M. (2017). Contaminación ambiental por ruido. Revista Médica Electrónica, 39(3), 640-649.

Bradfer-Lawrence, T., Gardner, N., Bunnefeld, L., Bunnefeld, N., Willis, S. G., \& Dent, D. H. (2019). Guidelines for the use of acoustic indices in environmental research. Methods in Ecology and Evolution, 10(10), 1796-1807. DOI: 10.1111/2041-210x.13254

Bustos, A. P. (2013). Paisaje sonoro en la ciudad de Bogotá: una aproximación a las características de frecuencia e intensidad del sonido en el espacio urbano (Tesis de pregrado). Pontificia Universidad Javeriana, Bogotá, Colombia. DOI: $10.1017 / C B O 9781107415324.004$

Cohen, M. A., \& Castillo, O. S. (2017). Ruido en la ciudad. Contaminación auditiva y ciudad caminable. Estudios Demográficos y Urbanos, 32(1), 65-96.

Deichmann, J. L., Hernández-serna, A., Delgado, A., Campos-Cerqueira, M., \& Aide, T. M. (2017). Soundscape analysis and acoustic monitoring document impacts of natural gas exploration on biodiversity in a tropical forest. Ecological Indicators, 74, 39-48. DOI: 10.1016/j.ecolind.2016.11.002

Do Nascimento, L. A., Campos-Cerqueira, M., \& Beard, K. H. (2020). Acoustic metrics predict habitat type and vegetation structure in the Amazon. Ecological Indicators, 117, 106679. DOI: 10.1016/j.ecolind.2020.106679

Fairbrass, A. J., Rennett, P., Williams, C., Titheridge, H., \& Jones, K. E. (2017). Biases of acoustic indices measuring biodiversity in urban areas. Ecological Indicators, 83, 169-177. DOI: 10.1016/j.ecolind.2017.07.064

Farina, A. (2013). Soundscape Ecology: Principles, Patterns, Methods and Applications. Dordrecht, Holland: Springer. DOI: 10.1007/978-94-007-7374-5

Farina, A., James, P., Bobryk, C., Pieretti, N., Lattanzi, E., \& McWilliam, J. (2014). Low cost (audio) recording (LCR) for advancing soundscape ecology towards the conservation of sonic complexity and biodiversity in natural and urban landscapes. Urban Ecosystems, 17(4), 923-944. DOI: 10.1007/s11252-014-0365-0

Fuller, S., Axel, A. C., Tucker, D., \& Gage, S. H. (2015). Connecting soundscape to landscape: Which acoustic index best describes landscape configuration? Ecological Indicators, 58, 207-215. DOI: 10.1016/j.ecolind.2015.05.057

Gasc, A., Pavoine, S., Lellouch, L., Grandcolas, P., \& Sueur, J. (2015). Acoustic indices for biodiversity assessments: Analyses of bias based on simulated bird assemblages and recommendations for field surveys. Biological Conservation, 191, 306-312. DOI: 10.1016/j.biocon.2015.06.018

Hernando, L., \& Orozco, R. (2015). Disponibilidad del recurso hídrico en la microcuenca del río Bermúdez. Región Central de Costa Rica. Observatorio Medioambiental, 18, 165-181. Recuperado de https://revistas.ucm.es/index.php/OBMD/article/viewFile/51289/47595

Hill, A. P., Prince, P., Piña Covarrubias, E., Doncaster, C. P., Snaddon, J. L., \& Rogers, A. (2017). AudioMoth: Evaluation of a smart open acoustic device for monitoring biodiversity and the environment. Methods in Ecology and Evolution, 9(5), 1199-1211. DOI: 10.1111/2041-210X.12955

Jeon, J. Y., \& Hong, J. Y. (2015). Classification of urban park soundscapes through perceptions of the acoustical environments. Landscape and Urban Planning, 141, 100-111. DOI: 10.1016/j.landurbplan.2015.05.005

Joo, W., Gage, S. H., \& Kasten, E. P. (2011). Analysis and interpretation of variability in soundscapes along an urban-rural gradient. Landscape and Urban Planning, 103(3-4), 259-276. DOI: 10.1016/j.landurbplan.2011.08.001

Liu, J., Kang, J., Luo, T., Behm, H., \& Coppack, T. (2013). Spatiotemporal variability of soundscapes in a multiple functional 
urban area. Landscape and Urban Planning, 115(2013), 1-9. DOI: 10.1016/j.landurbplan.2013.03.008

Mair, P., \& Wilcox, R. (2020). Robust statistical methods in R using the WRS2 package. Behavior Research Methods, 52(2), 464-488. DOI: 10.3758/s13428-019-01246-w

Matsinos, Y. G., Mazaris, A. D., Papadimitriou, K. D., Mniestris, A., Hatzigiannidis, G., Maioglou, D., \& Pantis, J. D. (2008). Spatio-temporal variability in human and natural sounds in a rural landscape. Landscape Ecology, 23(8), 945-959. DOI: 10.1007/s10980-008-9250-7

Ministerio de Salud. (14 de agosto de 2000). Reglamento para el Control de Contaminación por Ruido [N²8718-S]. Diario oficial La Gaceta $\quad$ N. $:$ : $155 . \quad$ Recuperado de https://www.seguridadpublica.go.cr/ministerio/gestion\%20ambiental/normativa\%20aplicable\%20y\%20vigente/d ecretos/D28718S.pdf

Nemeth, E., \& Brumm, H. (2010). Birds and anthropogenic noise: Are urban songs adaptive? American Naturalist, 176(4), 465-475. DOI: 10.1086/656275

Oro, M. I. (2017). Ecología Acústica (Tesis de grado). Universidad Politecnica de Madrid, Madrid, España.

Pandya, G. H. (2001). Urban noise-a need for acoustic planning. Environmental monitoring and assessment, 67(3), 379388.

Pavan, G; Scaravelli, D; Favaretto, A.; \& Macchio, S. (2015). Bioacoustics and Ecoacoustics Applied to Environmental. Rivista Italiana Di Acustica, 39(2), 68-74.

Pieretti, N., Duarte, M. H. L., Sousa-Lima, R. S., Rodrigues, M., Young, R. J., \& Farina, A. (2015). Determining temporal sampling schemes for passive acoustic studies in different tropical ecosystems. Tropical Conservation Science, 8(1), 215-234. DOI: 10.1177/194008291500800117

Pijanowski, B. C., Farina, A., Gage, S. H., Dumyahn, S. L., \& Krause, B. L. (2011). What is soundscape ecology? An introduction and overview of an emerging new science. Landscape Ecology, 26(9), 1213-1232. DOI: 10.1007/s10980-011-9600-8

Pijanowski, B. C., Villanueva-Rivera, L. J., Dumyahn, S. L., Farina, A., Krause, B. L., Napoletano, B. M., Gage, S. H., \& Pieretti, N. (2011). Soundscape Ecology: The Science of Sound in the Landscape. BioScience, 61(3), 203-216. DOI: 10.1525/bio.2011.61.3.6

Plan Regional Urbano de la Gran Área Metropolitana (PRUGAM). (2015). Análisis del territorio periurbano, actividades ecoturísticas y paisaje de la Gran Área Metropolitana de Costa Rica. Informe final. Universidad Nacional, Heredia, Costa Rica.

R Core Team (2019). R: A language and environment for statistical computing. R Foundation for Statistical Computing, Vienna, Austria. Recuperado de https://www.R-project.org/.

R Core Team (2020). R: A language and environment for statistical computing. R Foundation for Statistical Computing, Vienna, Austria. Recuperado de https://www.R-project.org/.

Ramalle-Gómara, E., \& Andrés de Llano, J. (2003). Utilización de métodos robustos en la estadística inferencial. Atención Primaria, 32(3), 177-182. Recuperado de https://www.elsevier.es/es-revista-atencion-primaria-27-articuloutilizacion-metodos-robustos-estadistica-inferencial-13049898

Ramírez-Alán, O. (2019). Sinax: Sound Index Analysis for Ecologist (version 1.3). Windows. Ubicación: R package. Version 1.3. Recuperado de https://rdrr.io/github/osoramirez/Sinax/

Salinas, E., \& Pérez, L. (2011). Procesos urbanos recientes en el Área Metropolitana de Concepción: transformaciones morfológicas y tipologías de ocupación. Revista de Geografia Norte Grande, 49, 79-97. DOI: 10.4067/S071834022011000200006. 
Shannon, G., McKenna, M. F., Angeloni, L. M., Crooks, K. R., Fristrup, K. M., Brown, E., Warner, K. A., Nelson, M. D., White, C., Briggs, J., McFarland, S., \& Wittemyer, G. (2016). A synthesis of two decades of research documenting the effects of noise on wildlife. Biological Reviews, 91(4), 982-1005. DOI: 10.1111/brv.12207

Sorensen, M., Barzetti, V., Keipi, K., \& Williams, J. (1998). Manejo de las áreas verdes urbanas. Washington D.C., Estados Unidos: División de Medio Ambiente del Departamento de Desarrollo Sostenible del Banco Interamericano de Desarrollo (BID). Recuperado de https://publications.iadb.org/es/publicacion/15940/manejo-de-las-areas-verdesurbanas

Sueur, J., Farina, A., Bobryk, C., Llusia, D., McWilliam, J., \& Pieretti, N. (2014). Ecology and acoustics: emergent properties from community to landscape. In Ecoacoustics. Ecology and acoustics: emergent properties from community to landscape. Paris, France: Muséum national d'Histoire naturelle

Sueur, J., Farina, A., Gasc, A., Pieretti, N., \& Pavoine, S. (2014). Acoustic indices for biodiversity assessment and landscape investigation. Acta Acustica United with Acustica, 100(4), 772-781. DOI: 10.3813/AAA.918757

Towsey, M., Wimmer, J., Williamson, I., \& Roe, P. (2014). The use of acoustic indices to determine avian species richness in audio-recordings of the environment. Ecological Informatics, 21(100), 110-119. DOI: 10.1016/j.ecoinf.2013.11.007

Ureña, N., Jiménez, F., Reynolds, J., \& Jones, J. (2005). Efectos del aumento poblacional. Recursos Naturales y Ambiente, $48,75-80$.

Welch, B. L. (1951). On the Comparison of Several Mean Values: An Alternative Approach. Biometrika, 38(3/4), 330. DOI: $10.2307 / 2332579$ 